\title{
Abstracts From the December 2020 Virtual International Sport + Exercise Nutrition Conference
}

Effects of Multi-Ingredient Pre-Workout Supplementation Across a 5-Day Resistance and Endurance Training Microcycle in MiddleAged Pre-Menopausal Women

Joel Puente-Fernández ${ }^{1}$, Marcos Seijo ${ }^{1}$, Eneko Larumbe-Zabala ${ }^{2}$, Alfonso Jiménez $^{3}$, Claire Rossato ${ }^{1}$, Fernando Naclerio ${ }^{1}$

${ }^{1}$ Department of Sports Science and Physical Education, Institute for Lifecourse Development, Centre for Chronic Illness and Ageing, University of Greenwich, UK

${ }^{2}$ School of Doctorate and Research, European University of Madrid, Spain

${ }^{3}$ University of Sheffield Hallam University, UK

Pre-workout multi-ingredient admixtures are currently used to maximise subsequent exercise performance. The present double-blind, cross-over study compared the acute effects of ingesting a multi-ingredient (PREW) vs. maltodextrin (CHO) over a training week (microcycle) composed by three resistance training (RT) workouts alternated with two 30-min lowintensity endurance sessions (END) on total volume lifted $(\mathrm{kg})$ during RT and substrate oxidation during END. Additionally, post-workout decreases of involuntary and voluntary muscle function, subjective measures of effort and feelings were analysed. Following a baseline performance assessment, seven recreationally trained middle-aged women ( $49 \pm 4$ years old) completed two identical microcycles separated by a 2-week washout period while receiving either PREW or $\mathrm{CHO}$ (15 minutes prior to workout). The exercise volume (kg lifted), per session (WVOL) and for the total three RT (WeeklyVol) was calculated. Fatty acid oxidation (FAO) during 30-min cycling corresponding to their individually-determined maximal fat oxidation was measured using expired gasses and indirect calorimetry. Assessments of strength and power performance, along with tensiomyography analysis, were conducted within $20 \mathrm{~min}$ after each RT. Under the PREW, the participants completed significantly $(p=.001)$ higher WeeklyVol and WVOL (for the three RT) and higher, but not significant FAO ( $p=.17 ; \mathrm{d}=0.6)$ during the first END workout. No other statistically significant differences were observed between conditions. Compared to $\mathrm{CHO}$, a pre-workout multi-ingredient appears to increase resistance exercise volume and may suppose a tendency to favour fat oxidation during low-intensity endurance exercises.

Factors Mediating the Effect of Caffeine Supplementation on the IL-6 and IL-10 Response to a Treadmill Exercise Test

Ll Rodas, S Martínez, A Aguiló, P Tauler

Research Group on Evidence, Lifestyles and Health, University of the Balearic Islands, Spain

Caffeine is one of the most consumed ergogenic aids used by athletes. It has been previously reported that caffeine could modify the response of two essential cytokines, IL-6 and IL-10, to an acute bout of exercise. The aim of this study was to determine whether adrenaline, cortisol or cyclic adenosine monophosphate (cAMP) mediate this effect. In a randomized, crossover, double-blind study, thirteen healthy, well-trained amateur male athletes performed a treadmill exercise test (60 minutes at 70\% VO2max), on two different occasions, after ingesting $6 \mathrm{mg} / \mathrm{kg}$ body mass of caffeine or a placebo. Blood samples were taken before and immediately after exercising, as well as two hours after finishing the exercise. The plasma concentrations of IL-10, IL-6, adrenaline, cortisol and cAMP were determined, and multiple linear regression models for IL-10 and IL-6 were analysed. The independent variables considered were time (pre-exercise, post-exercise, recovery), condition (caffeine, placebo) and adrenaline, cortisol and cAMP concentrations. The IL-10 model also included IL-6 as an independent variable. Caffeine supplementation influenced IL-6 $(3.04 \pm 0.40$ vs. $3.89 \pm 0.62 \mathrm{pg} / \mathrm{mL}, p=.003)$ and IL-10 (2.42 \pm 0.54 vs. $3.47 \pm 0.72 \mathrm{pg} / \mathrm{mL}, p=.01$ ) levels, with higher concentrations after exercise in the supplemented condition. The regression analysis showed that IL-6 was the main predictor for IL-10 plasma concentrations (R2 change $0.435, p<.001$ ); time (R2 change $0.083, p=.001$ ) and cAMP ( $\mathrm{R} 2$ change $0.051, p=.004$ ) were also found to be significant predictors. Adrenaline was found to be the main predictor for IL- 6 concentrations (R2 change $0.379, p<.001)$; with the time factor also being a significant predictor (R2 change $0.213, p<.001$ ). In conclusion, the results of this study point to IL-6 as the main factor for determining IL-10 response to exercise after caffeine supplementation. In turn, the effects of caffeine on the IL-6 response to exercise seem to be mainly dependent on adrenaline.

Sports Nutrition Knowledge Among Polish Athletes According to the Abridged Nutrition for Sport Knowledge Questionnaire (A-NSKQ)

B Szczepańska ${ }^{1}$, O Surała $^{1}, \mathrm{~J}$ Orysiak $^{2}, \mathrm{~J}$ Malczewska-Lenczowska ${ }^{1}$

${ }^{1}$ Department of Nutrition Physiology and Dietetics, Institute of SportNational Research Institute, Warsaw, Poland

${ }^{2}$ Department of Ergonomics, Central Institute for Labour ProtectionNational Research Institute, Warsaw, Poland

A well-planned nutrition strategy may promote training adaptations. Higher levels of sports nutrition knowledge may translate to better diet quality, increasing athletes' chances of success. Determining the level of nutritional knowledge in athletes can identify both areas for education and specific sports groups in need of educational intervention. In the study on 128 Polish athletes (77 males, 51 females, age: 17-32 y.), the nutrition knowledge was measured with the validated A-NSKQ. The A-NSKQ measures both general nutrition knowledge (GNK) and sport nutrition knowledge (SNK). Based on normality, the parametric t-test, or nonparametric Spearman correlation, the U-Mann-Whitney test were used. Significance was set at an alpha level of $p \leq .05$. In the whole group, the mean total A-NSKQ score was $44 \pm 12 \%$, which was classified as "poor." The score of SNK was significantly lower than GNK $(37 \pm 14 \%$ vs. $53 \pm 15 \% ; p<.001)$. There were no differences between male and female athletes in A-NSKQ total, GNK, and SNK score. The total A-NSKQ and SNK score were positively correlated to age $(\mathrm{R}=0.27, p=.002$; $\mathrm{R}=0.29, \quad p=.001 ;$ respectively), and the level of education $(\mathrm{R}=0.31, p<.001 ; \mathrm{R}=0.37, p<.001$; respectively). When stratified into two sport categories, individual-sport athletes got higher total $(47 \pm 12 \%$ vs. $40 \pm 13 \%, p=.001)$, GNK $(56 \pm 14 \%$ vs. $49 \pm 16 \%$, $p=.022)$ and SNK score $(40 \pm 14 \%$ vs. $31 \pm 13 \%, p=.001)$ than 
team-sport athletes. Our results indicate the low level of nutritional knowledge in Polish athletes and, in particular, in individuals from team sports. The findings indicate the need to educate Polish athletes, particularly regarding current recommendations in sports nutrition.

\section{An Investigation Into Changes in Cognitive Function of Irish Amateur Boxers as They Approach Their Weight Category Weight}

\author{
L Mahony $^{1,2}$, SM Madigan ${ }^{2}$, BV O’Neill ${ }^{3}, \mathrm{C} \mathrm{Blakely}^{1}$, AM McNeilly ${ }^{1}$ \\ ${ }^{1}$ Sport and Exercise Sciences Research Institute, Ulster University, \\ Newtownabbey, Co. Antrim, BT37 0QB \\ ${ }^{2}$ Sport Ireland Institute, Abbottstown, Dublin 15 \\ ${ }^{3}$ GSK Human Performance Lab, Brentford, United Kingdom
}

Boxing is a combat sport which typically sees athletes competing at the lightest bodyweight possible to gain a competitive advantage. Several methods are used to reduce bodyweight and traditionally dehydration has played a significant role in rapid weight-loss. These rapid weight-loss methods have been shown to result in decrements in cognitive performance and can impact both short and long-term health and sports performance. 8 members of the Irish High-Performance boxing programme who were in the process of 'making-weight' participated in this research. Baseline hydration, body weight, skinfolds, (sum of 7), and cognitive assessments (simple and choice reaction time, attention, learning and memory) were completed six weeks prior to a targeted competition. These measurements were then repeated in the week of the targeted competition, as participants were 'making-weight'. There were statistically significant changes in bodyweight $(n=8)$, and in the corresponding weight above weight category, but no statistically significant changes in skinfolds $(n=5)$ or hydration, $(n=8)$ over the 6-week training block. At baseline, mean hydration levels were $800 \mathrm{mOsm} \cdot \mathrm{kg}^{-1}$ and this increased to $>850 \mathrm{mOsm} \cdot \mathrm{kg}^{-1}$ over the 6 weeks. There were no statistically significant changes found in the cognitive function measurements. Due to the wide variation in hydration levels, it cannot be ascertained if hydration status affected reaction times in this study. There is also the element of habitual dehydration that boxers go through whilst making weight, further research is warranted to see if the body does adapt to these situations. Even though there were no statistically significant changes found in simple and choice reaction time speeds; from a practical application, coaches may be interested to note that 6 of the 8 boxers were slower in both of these elements as they came closer to their targeted competition. This warrants further investigation to determine, if weight making practices were improved, could reaction times also improve.

\section{Alkaline Diet Improves Vertical Jump Performance in Volleyball Players}

H Eryiğit ${ }^{1,2}$, B Ramanli ${ }^{1}$, H Turnagöl ${ }^{2}$

${ }^{1}$ Faculty of Sport Sciences, Pamukkale University Denizli, Turkey

${ }^{2}$ Faculty of Sport Sciences, Hacettepe University, Ankara, Turkey

Increased buffering capacity helps to maintain muscle contraction and delay muscle fatigue during high intensity exercise. In this regard various nutritional supplements increasing buffering capacity are used to improve high intensity exercise performance. In recent years, it has been postulated that an alkaline diet may also increase the exercise performance by creating a buffering effect. Therefore, the aim of the current research is to compare the acute effects of alkaline (ALD) and acidic diets (ACD) on anaerobic performance and hydration level in volleyball players. Eleven semiprofessional male volleyball players (age $=20 \pm 1 \mathrm{y}$, training age $=9 \pm 3$ y) voluntarily participated in the study in a randomized, single-blind, cross-over design. Each participant visited the laboratory three times: baseline (B) measurements were taken with their unmodified usual diet, followed by two measurements following a 4-day ACD or ALD program with a 1-week washout period between. ACD and ALD contents were calculated individually and the participants were provided with packed daily meals. Measured variables included urine $\mathrm{pH}, 30 \mathrm{sec}$ Wingate anaerobic exercise performance, countermovement jump (CMJ) and squat jump (SJ) performances, and blood lactate (La). Repeated measures ANOVA was used to analyse the data. Bonferroni correction was used as post-hoc analysis. No main effect of diet was found on Wingate anaerobic exercise performance $(p>.05)$. However, both SJ and CMJ performance increased significantly with the alkaline diet $(\mathrm{CMJ}(\mathrm{cm})$; ALD $40.7 \pm 6.4$, ACD 39.9 \pm 5.9 , baseline $38.9 \pm 6.5, p<.05 ; \mathrm{SJ}(\mathrm{cm})$; ALD $37.6 \pm 5.7, \mathrm{ACD} 36.3 \pm 5.3$, baseline $35.4 \pm 6.0, p<.05$ ). Hydration level (ALD $1017 \pm 4$, ACD $1024 \pm 4$, baseline $1019 \pm 4.9, p<.05$ ) and urine $\mathrm{pH}$ (ALD $6.7 \pm 0.5$, ACD 5.1 \pm 0.3 , baseline $5.2 \pm 0.4, p<.05$ ) were significantly different in favour of the ALD while no difference was found between BLa levels (mmol/L, ALD 6.2 \pm 2.0 ACD 6.2 \pm 1.3 , baseline $6.8 \pm 1.8, p>.05$ ). As a result; volleyball players may be advised to have an alkaline diet starting four days before the matches. An alkaline diet can be an easy and natural way to improve hydration and increase vertical jump performance for athletes. However, more studies are needed to elucidate the effect of alkaline diet on anaerobic performance.

\section{Estimation of Athlete's Prevalence of Risk of Low Energy Availability at the 26th European Cross-Country Championships}

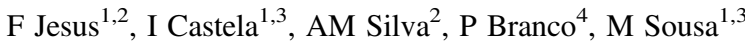

${ }^{1}$ Nutrition \& Metabolism, NOVA Medical School I Faculdade de Ciências Médicas, Universidade NOVA de Lisboa, Lisboa, Portugal

${ }^{2}$ Exercise and Health Laboratory, CIPER, Faculdade de Motricidade Humana da Universidade de Lisboa. Estrada da Costa, 1499-688 CruzQuebrada, Portugal

${ }^{3}$ CINTESIS, NOVA Medical School, NMS, Universidade Nova de Lisboa, 1169-056 Lisboa, Portugal

${ }^{4}$ Medical \& Anti-doping Commission, European Athletics, Lausanne, Switzerland

Energy availability (EA) refers to the difference between energy intake and exercise energy expenditure divided by fat-free mass. Low EA (LEA) is linked with impaired physiological functioning such as menstrual function and bone health. Cross-country (CC) is a weight sensitive sport with high training loads and physiological demands. The risk of LEA in elite CC athletes is considered high but literature is still limited. The aim of this study was to estimate the prevalence of elite European CC athletes at risk of LEA and the presence of LEA related negative outcomes. Athletes $\geq 18 \mathrm{y}$ competing at the 26th European CC Championships were invited to fil the LEA in Females Questionnaire (LEAF- Q). The LEAF-Q is a validated survey that identifies female athletes at risk of LEA. It has 3 sections: (i) injuries; (ii) gastrointestinal function; (iii) menstrual function and use of contraceptives. The LEAF- Q has also been applied in male athletes. A total of 207 valid surveys were collected ( 83 females, $22 \pm 4$ y) plus 16 excluded. A high prevalence of athletes at risk of LEA $(64 \%)$ was observed, with a higher percentage in women than men $(80 \%$ and $54 \%, p<.001)$. During the previous year, $52 \%$ reported missing training or competition due to injuries. Furthermore, 10 athletes $(\%)$ reported stress fractures. For gastrointestinal function, 54\% reported evacuating once a week or more rarely. Regarding menstrual function, $43 \%$ reported having their first menstruation at 15 years or older, while $4 \%$ reported never having menstruated. Additionally, more than $40 \%$ reported not having normal menstruation while $50 \%$ reported changes of menstruation with training. Furthermore, athletes reported using contraceptive either to regulate menstrual cycle in relation to performance $(n=7)$ or to prevent the cessation of menstruation $(n=3)$. In 
sum, elite $\mathrm{CC}$ athletes are in high risk of LEA. Although, in the past, LEA might have been interpreted as a usual (and needed) procedure, nowadays it is recognised that it may lead to several negative consequences. Therefore, these athletes should be followed by multidisciplinary teams to prevent, treat, or minimize LEA and its effects. Moreover, educational programmes targeting athletes and their support personnel should be developed to prevent and increase awareness and knowledge on LEA.

Consuming Insect vs. Beef-Derived Protein Alters Postprandial Plasma Aminoacidemia, but has Little Effect on Appetite Hormones, Subjective Appetite Sensations

$\mathrm{J} \mathrm{Dai}^{1}{ }, \mathrm{~J}$ Lov $^{1}$, P Martin ${ }^{1}$, A Gritsas ${ }^{2}$, TA Churchward-Venne ${ }^{1,2,3}$

${ }^{1}$ Department of Kinesiology and Physical Education, McGill University, Canada

${ }^{2}$ Research Institute of the McGill University Health Centre, Canada

${ }^{3}$ Division of Geriatric Medicine, McGill University, Canada

Higher protein, energy-restricted diets containing $1.2-1.6 \mathrm{~g}$ protein $\cdot \mathrm{kg}^{-1}$. $\mathrm{d}^{-1}$, support greater weight loss, fat loss, and preservation of lean mass compared to normal protein diets. The effectiveness of these diets may be related to modulation of appetite and satiety, leading to reductions in energy intake. However, types of dietary protein may differ in their effects on appetite and satiety. The objective of this study was to examine the acute effects of consuming macronutrient-matched beverages containing $25 \mathrm{~g}$ protein from crickets or beef on postprandial plasma glucose, insulin, and amino acid concentrations, subjective appetite sensations, and ad libitum energy intake. In a randomized, double-blind, crossover study, 20 young men (age: $23 \pm 1 \mathrm{y}$ [mean $\pm \mathrm{SEM}]$ ) completed two test days during which arterialized blood samples and VAS questionnaires were obtained at baseline, and over 5-h following cricket or beef protein ingestion to assess postprandial plasma glucose, insulin, and amino acid concentrations, and subjective appetite sensations. An ad libitum test meal was provided after each test day to assess energy intake. Postprandial net incremental area under the curve (niAUC) was greater for cricket vs. beef protein for plasma leucine (cricket: $11033 \pm 1601$; beef: $3621 \pm 934 \mu \mathrm{mol} / \mathrm{L} \times 300 \mathrm{~min}$ ), branched chain amino acid (BCAA) (cricket: $35325 \pm 3364$; beef: $12263 \pm 2503 \mu \mathrm{mol} / \mathrm{L} \times 300 \mathrm{~min}$ ), and essential amino acid (EAA) (cricket: $59610 \pm 5311$; beef: $24101 \pm 4711 \mu \mathrm{mol} /$ $\mathrm{L} \times 300 \mathrm{~min}$ ) concentrations (all $p<.05$ ). Postprandial niAUC sensations of 'hunger' were lower following beef vs. cricket protein (beef: $-20 \pm 733$; cricket: $1564 \pm 714 \mathrm{~mm} \times 300 \mathrm{~min})(p=.042)$ but were not different for 'fullness', 'desire to eat', or 'prospective food consumption' (all $p>.05$ ). Participants consumed $1030 \pm 46 \mathrm{kcal}$ during the ad libitum meals, with no difference between protein sources $(p=.277)$. Postprandial aminoacidemia differs following cricket and beef protein, with a greater niAUC for leucine, BCAA, and EAA concentration with cricket protein. Subjective 'hunger' was lower with beef protein; however, all other appetite sensations and ad libitum energy intake were similar between proteins. Cricket protein may represent a novel source of dietary protein when developing higher-protein meals to support appetite regulation.

Influence of Macronutrient Intake on Different Estimates of Energy Availability in a Sample of Male National Team Athletes

CI Ayala-Guzmán ${ }^{1}$, I Castillo-Rosales ${ }^{2}$, K López-Flores ${ }^{2}$, D FrancoMárquez $^{2}$, M Ortega-Cortes ${ }^{2}$, O Nájera-Medina ${ }^{3}$, R Bojalil-Parra ${ }^{3}$, L Ortiz-Hernández ${ }^{3}$

${ }^{1} \mathrm{PhD}$ student in Biological and Health Sciences, Universidad Autónoma Metropolitana, Mexico

${ }^{2}$ Research assistant, Universidad Autónoma Metropolitana, Mexico

${ }^{3}$ Professor-Researcher, Universidad Autónoma Metropolitana, Mexico
The influence of macronutrient intake on energy availability (EA) has been reported, but, since there's no standardized method to estimate EA, it's not clear if there are significant differences in the contribution of macronutrient intake on EA. The aim of this study was to identify the influence of macronutrient intake on EA, analyzing differences between methods for estimating EA. Athletes affiliated with the Mexican Olympic Committee were recruited. A convenience sample of 10 male athletes (mean age $=24 \pm 4$ y) participated in the study. Body composition was measured by anthropometry (ANT), bioelectrical impedance analysis (BIA), and dual-energy X-ray absorptiometry (DXA). Energy consumption and heart rate were recorded for a week. Using linear regression models, energy expenditure during exercise was predicted by heart rate monitoring and from the $\mathrm{VO} 2$ and $\mathrm{VCO} 2$ during a laboratory exercise test. EA was estimated using ANT (EA-ANT), BIA (EA-BIA), and DXA (EA-DXA). Correlations between energy intake from macronutrients were lower with EA-BIA, but higher with EA-ANT and EA-DXA. Body- weight-relative energy intake $(\mathrm{kcal} / \mathrm{kg}$ ) from macronutrients, compared to total energy intake (kcal), had stronger correlations with EA. Protein intake (kcal/kg) had the strongest correlations with EA estimates $($ EA-ANT $=0.79$, EA$\mathrm{BIA}=0.65$, EA-DXA $=0.75, p<.05)$. In linear regression models, when considering EA as a dependent variable and body-weight-relative energy intake from macronutrients as independent variables, it is shown that the accuracy was higher, and the standard error of estimates was lower. Body-weight-relative energy intake from proteins was the most accurate predictor of EA $(\mathrm{EA}-\mathrm{ANT}=\mathrm{R} 2=63.1 \%$; EA-BIA $=\mathrm{R} 2=42.9$; EADXA $=55.9 \%$ ), followed by body-weight-relative energy intake from carbohydrates $($ EA-ANT $=$ R2 $=58.1 \%$; EA-BIA $=43.0 \%$; EA-DXA $=$ $53.3 \%$ ). In contrast, after including protein and carbohydrate energy intake in the same model, none of the regression coefficients were associated with EA. In conclusion, compared to total energy intake, body-weight relative energy intake from macronutrient influence EA to a greater extent. Finally, body-weight-relative energy intake from protein seems to influence on EA to a greater extent than carbohydrates, regardless of the method of estimating EA

Correlation, Agreement, and Accuracy of Different Methods for Estimate Energy Availability in a Sample of Male National Team Athletes

CI Ayala-Guzmán ${ }^{1}$, I Castillo-Rosales ${ }^{2}$, K López-Flores ${ }^{2}$, D FrancoMárquez $^{2}$, M Ortega-Cortes ${ }^{2}$, O Nájera-Medina ${ }^{3}$, R Bojalil-Parra ${ }^{3}$, L Ortiz-Hernández ${ }^{3}$

${ }^{1} \mathrm{PhD}$ student in Biological and Health Sciences, Universidad Autónoma Metropolitana, Mexico

${ }^{2}$ Research assistant, Universidad Autónoma Metropolitana, Mexico

${ }^{3}$ Professor-Researcher, Universidad Autónoma Metropolitana, Mexico

In recent years the importance of energy availability (EA) in the health and physical performance of athletes has been reported. Nevertheless, there is no standard methodology for its estimation. Therefore, this can lead to differences in estimates of low-EA between samples of athletes. This study aimed to know the correlation, agreement, and accuracy between estimates of EA. Athletes affiliated with the Mexican Olympic Committee were recruited. A convenience sample of 10 male athletes (mean age $=24 \pm 4 \mathrm{y}$ ) participated in the study. Body composition was measured by anthropometry (ANT), bioelectrical impedance analysis (BIA), and dual-energy x-ray absorptiometry (DXA). Fat-free mass (FFM) was calculated from the difference of weight and body fat mass estimated by three anthropometric prediction equations: Evans, Faulkner, and Carter. Energy consumption and heart rate were recorded for a week. Using linear regression models, energy expenditure during exercise was predicted by heart rate monitoring and from the $\mathrm{VO} 2$ and $\mathrm{VCO} 2$ during a laboratory exercise test. EA was 
estimated using ANT (EA-ANTEvans, EA-ANTFaulkner, EA-ANTCarter), BIA (EA-BIA), and DXA (EA-DXA). EA ranged from 30.3 to $35.5 \mathrm{kcal} /$ $\mathrm{kgFFM} / \mathrm{d}$. Compared to EA-DXA, EA-BIA and EA-ANTFaulkner showed a very high correlation $(0.986$ and $0.974, p<.050)$ and agreement $(0.976$ and $0.935, p<.050)$. EA-BIA was the most accurate predictor of EA-DXA and showed the lowest mean absolute percentage error $(\mathrm{R} 2=97.2 \%$, SEE $=$ 1.50, MAPE $=13.1 \%)$, followed by EA-ANTFaulkner $(\mathrm{R} 2=94.8 \%$, SEE $=2.04, \mathrm{MAPE}=11.4 \%)$. All methods underestimate EA $(2.1$ to $5.2 \mathrm{kcal} /$ $\mathrm{kgFFM} / \mathrm{d}$ ), and Bland-Altman graphs made evident that the mean bias of EABIA ( $2.1 \mathrm{kcal} / \mathrm{kgFFM} / \mathrm{d})$ was lower than EA-ANTFaulkner ( $4.0 \mathrm{kcal} / \mathrm{kgFFM} /$ d). Nevertheless, no clear trend on the underestimation of EA was observed. In conclusion, BIA is a more accurate method for the estimation of EA. But, when the BIA method is not feasible, compared to Evan's and Carter's equations, Faulkner's equation is recommendable for estimating body fat mass, and subsequently fat-free mass, in order to estimate EA of highly trained athletes. Future research should focus on analyze differences between other anthropometric predictive equations, and also different estimates of total energy intake and energy expenditure during exercise.

\section{Ketone Monoester Supplementation Does Not Expedite the Recovery} of Indices of Muscle Damage After Eccentric Exercise

$\mathrm{J} \mathrm{Lov}^{1}$, PW Martin-Arrowsmith ${ }^{1}$, J Dai ${ }^{1}$, JA Morais ${ }^{1,2,3}$, TA ChurchwardVenne $^{1,2,3}$

${ }^{1}$ Department of Kinesiology and Physical Education, McGill University, Montreal, Quebec, Canada

${ }^{2}$ Division of Geriatric Medicine, McGill University, Montreal, Quebec, Canada

${ }^{3}$ Research Institute of the McGill University Health Centre, Montreal, Quebec, Canada

Exogenous ketone supplements can increase circulating b-hydroxybutyrate $(\beta-\mathrm{OHB})$ to millimolar concentrations within minutes. In addition to serving as an alternative fuel substrate, $\beta$-OHB is a signaling molecule that may regulate muscle protein turnover, inflammation, and oxidative stress. Therefore, ketone supplements and elevated $\beta$-OHB may impact postexercise recovery. The purpose of this study was to evaluate the effects of a ketone monoester supplement on indices of muscle damage during recovery after eccentric exercise. In a randomized, double-blind, independent group design, 20 healthy moderately active young adults consumed $360 \mathrm{mg} \cdot \mathrm{kg}^{-1}$ bodyweight of a ketone monoester (KET) or energy-matched carbohydrate (CON) supplement twice daily following eccentric exercise (drop jumps). Maximal isometric voluntary contraction (MIVC) torque, counter-movement jump (CMJ) height, and muscle soreness via visual analogue scale (VAS) and pressure pain threshold (PPT) were measured before (PRE), and immediately (POST), $24 \mathrm{~h}$, and $48 \mathrm{~h}$ post-exercise. Blood samples were collected for analysis of $\beta-\mathrm{OHB}$, creatine kinase $(\mathrm{CK})$, and select pro- and anti-inflammatory cytokines. Peak blood $\beta-\mathrm{OHB}$ concentration after supplement intake was greater $(p<.001)$ in KET $(4.4 \pm 0.8 \mathrm{mM})$ vs. CON $(0.4 \pm 0.3 \mathrm{mM})$. Serum CK concentration increased (time; $p<.001)$ at $24 \mathrm{~h}\left(319 \pm 315 \mathrm{IU} \cdot \mathrm{L}^{-1}\right)$ and $48 \mathrm{~h}(198 \pm$ $\left.155 \mathrm{IU} \cdot \mathrm{L}^{-1}\right)$ after exercise compared to PRE $\left(136 \pm 122 \mathrm{IU} \cdot \mathrm{L}^{-1}\right)$, with no difference between KET and CON. Exercise led to a similar early reduction in MIVC (KET: $-19.9 \pm 14.6$; CON: $-22.6 \pm 11.1 \%$ ) and CMJ (KET: $-11.0 \pm 7.5$; CON: $-13.0 \pm 8.7 \%)$ at POST relative PRE. However, there was no difference between KET and CON on the recovery of MIVC at $24 \mathrm{~h}$ (KET: $-15.4 \pm 20.4$; CON: $-18.7 \pm 20.1 \%$ ) or 48 h (KET: $-7.2 \pm 21.2$; CON: $-11.8 \pm 20.2 \%$ ), or CMJ at 24 h (KET: $-9.2 \pm 11.5$; CON: $-13.4 \pm$ $10.8)$ or 48 h (KET: $-12.5 \pm 12.4$; CON: $-9.1 \pm 11.7)$. Measures of muscle soreness based on VAS and PPT increased during recovery after eccentric exercise (time, both $p<.001$ ), with no difference between KET and CON on either measure. Blood MCP-1 was greater (group: $p=.007$ ) in CON
$(236 \pm 11 \mathrm{pg} / \mathrm{mL})$ vs. KET $(187 \pm 11 \mathrm{pg} / \mathrm{mL})$. Twice daily ingestion of a ketone monoester does not enhance the recovery of indices of muscle damage after eccentric exercise in moderately active, healthy young adults.

\section{Impact of National Coronavirus Restrictions on Dietary Patterns and Training Schedules of German Competitive Athletes}

A Carlsohn, CD Rossi, N Riedel, S Adam

Department of Nutrition and Home Economics, Faculty of Lifes Sciences, University of Applied Sciences Hamburg, Germany

In spring 2020 coronavirus (CV) restrictions influenced our everyday life. Besides different $\mathrm{CV}$ rules (e.g., prohibition to use public sports facilities, closure of restaurants and canteens) panic buying of food was observed in Germany. Purpose was to analyse the impact of national CV restrictions on food intake, motivation for healthy eating and on individual training schedule of German competitive athletes. Data of 357 competitive athletes (97 recreational, 80 national squad, 48 youth elite, 19 master, 113 with missing specification) were captured 04/2020 until 09/2020 using an online survey. Intake of 15 food categories was assessed using four answering options (consumed more OR less OR equal amounts before and during CV restrictions OR I do not consume this food). Changes in exercise and motivation were assessed with multiple answer items. First data are analysed descriptively. Most athletes followed a mixed diet $(68 \%), 11 \%$ were vegetarians and $3 \%$ vegans. The majority of athletes $(84 \%)$ was not able to exercise according to the schedule, $20 \%$ reported a reduced training volume and $37 \%$ had to change key sessions. Only $10 \%$ exercised at their common training facility while $20 \%$ exercised predominantly at home. Regarding food intake $(\mathrm{N}=76)$ the majority of athletes reported equal amounts in most food categories before and during $\mathrm{CV}$ restrictions. Exceptions include increased intakes of vegetables (by $41 \%$ of athletes), fruits (37\%), sweets and salty snacks $(30 \%)$ and alcoholic drinks $(27 \%)$. The pandemic did not influence the use of supplements. $50 \%$ of athletes were more motivated to try new recipes during $\mathrm{CV}$ restrictions, and $45 \%$ felt more motivated to prepare healthy food. $35 \%$ were more motivated to apply sports nutrition recommendations during CV restrictions. $95 \%$ of athletes did not report requirements for dietary counselling. During CV restrictions the majority of German athletes had to change training schedules. The pandemic did not substantially change food intake for most food categories including supplements. However, a trend for increased intake of fruits and vegetables, sweets and alcoholic drinks was reported. Even though athletes did not demand nutritional guidance, professional counselling might support athletes to adapt food intake to the changed exercise schedule and promote healthy eating.

\section{Personality Traits of Supplement Users Versus Non-Supplement Users}

T Simper ${ }^{1}$, A Chappell ${ }^{2}$

${ }^{1}$ Sheffield Hallam University, Department of Food and Nutrition, Pond Street, Sheffield South Yorkshire, S1 1WB, United Kingdom

${ }^{2}$ Robert Gordon University, School of Pharmacy and Life Science, Robert Gordon University, Garthdee Road, Aberdeen, AB10 7AQ, United Kingdom

Openness to experience, Conscientiousness, Extraversion, Agreeableness and Neuroticism are agreed upon by many psychologists as the 'Big 5' personality traits and are correlated with numerous life outcomes such as career success and life-satisfaction. We surveyed 585 people from a snowball sample on their levels of physical activity, using the International Physical Activity Questionnaire (IPAQ) and their personality traits using the ten Item Personality Index (TIPI) their supplement use and selfreported BMI. The sample was then subdivided into non-supplement 
users, endurance supplement users and bodybuilding supplement users. The sample was 30\% (N173) male and 84\% (N 489) white and 10\% (N 61) Asian/Asian British. Subjects were mean age $34\left( \pm 8\right.$ y) BMI $26\left( \pm 7 \mathrm{~kg} / \mathrm{m}^{2}\right)$. Logistic regression was employed in two models, firstly to assess the impact of the 5 personality traits on influencing being a supplement user and secondly to assess these personality factors influencing the subdivision of 'bodybuilding' supplement users versus 'endurance' supplement users. For users versus non-users only conscientiousness was significantly predictive of being a supplement user ( $p=.025)$ OR 1.17 (95\% CI 1.020-1.349) indicating for every unit of conscientiousness a subject scored higher there was a .16 increase $(16 \%)$ in likelihood of being a supplement user. For the bodybuilding versus endurance supplement classifications the only variable which predicted being an endurance supplement user was agreeableness $(p=.018)$ OR $1.3495 \%$ CI 1.051.70. Indicating as agreeableness rose by a unit likelihood of being one of the endurance supplement users also rose by 0.34 or $34 \% 95 \% \mathrm{CI}=1.05-$ 1.70. Previous work has highlighted the impact of conscientiousness on various domains such as career success and weight management and other work has also suggested more anti-social traits amongst bodybuilders which may align with the finding of lower levels of agreeableness amongst this group. We interpret the greater conscientiousness in users of supplements as an indication of generally being more concerned with personal health. Findings relating to endurance versus bodybuilding supplement use need to be interpreted with caution and warrant further investigation.

Sources of Nutrition Information and Nutrition Knowledge of Female and Male Rowers in Traditional Rowing (Fixed Bank Rowing)

\section{H Albizu-Yeregui}

Department of Physical Education and Sports, University of Basque Country (UPV- EHU), Spain

Adequate nutrient intake is important to optimize a good health and to increase sports performance, but it is observed that it is a factor that many athletes overlook. The reason could be related to low levels of nutrition knowledge (NK) and the use of low-quality nutritional information sources. A total of 73 rowers (52 male and 21 female) in traditional rowing participated in the study, whose aim is to know the importance they give to nutrition, which are their source of nutrition information and to their NK evaluation. For this evaluation, a developed and validated by Reilly and Maughan questionnaire was used. We omitted the question of demographics, dietary behaviours and the specific swimmers' section. Finally, the questionary is made up of 59 questions distributed in 7 topics (hydration, weight control, dietary supplements, general nutrition, sports nutrition, protein, strategies for training and food choices). On the other hand, they were asked 5 questions about sources of nutrition information and general question about it. All participants answered that nutrition is very important for athletic performance, but $47 \%$ of participants reported that they had the necessary NK and $30 \%$ poor, similar data to those found literature. The survey revealed that the Dietitian-nutritionist is the number one source of nutrition information followed by Media, Internet, Friends, Doctors, Trainer and Family. Each question in the questionnaire has a single correct answer that when selected is counted as a right guess. The $51 \%$ of the responses to the questionnaire were correct and the other $49 \%$ were incorrect or they were not sure, no differences between sexes. It is observed that a group of men group that have a higher percentage $(55 \%)$ of correct answer and is the one that has a dietitian-nutritionist in the club. These data are far from the threshold established by Torre-McGehee which is $75 \%$ correct to say that they have adequate knowledge about nutrition. The areas of weight control, dietary supplements, and general nutrition had the worst scores with $43 \%$, $33 \%$ and $47 \%$ respectively. Nutrition being so important, we observed that there is a lack of NK in our athletes because knowledge is often not acquired from reliable sources that know the subject well.

\section{Identification of Risk Groups for Low Bone Stiffness in German School Children}

J Heydenreich, A Schweter, P Lührmann

Institute of Health Sciences, University of Education Schwäbisch Gmünd, Germany

A low bone density during childhood and adolescence is one important risk factor for osteoporosis development in later life. Besides genetics, several lifestyle factors seem to play a central role for achievement of bone mass during growth. Identification of risk groups for low bone stiffness index (SI) might be one important strategy for osteoporosis prevention. Purpose was to identify risk groups for low bone SI in school children. In 248 girls $\left(13.4 \pm 1.9\right.$ years, BMI: $\left.20.2 \pm 4.8 \mathrm{~kg} / \mathrm{m}^{2}\right)$ and 231 boys $(13.6 \pm 1.7$ years, BMI: $19.3 \pm 3.3 \mathrm{~kg} / \mathrm{m}^{2}$ ) the following parameters were assessed: calcaneal SI (quantitative ultrasound), body composition (bioelectrical impedance analysis), food intake (food frequency questionnaire), total energy expenditure (TEE) and daily duration of sports (standardized questionnaire), and estimated resting metabolic rate (RMR; Müller et al. 2004). Physical activity level (PAL) was calculated by dividing TEE with RMR. Individual food intake of 8 food groups (fruits/vegetables, fish, bread, milk/dairy products, meat/sausages, tolerated food, soft drinks, caffeinated beverages) was related to the German recommendations (Bone Healthy Eating Index, BoneHEI; 0-100 points). Participants were classified according to age- and sex-specific tertiles of SI (low, medium and high SI). Between-group comparisons were achieved by Kruskal-Wallis $H$ tests $(\alpha=0.05)$. Girls from the lowest tertile of SI had a significantly lower body mass $(49.2 \pm 16.7$ vs. $54.8 \pm 12.2 \mathrm{~kg} ; p<.01)$, BMI $(19.6 \pm 5.4$ vs. $21.3 \pm$ $\left.3.9 \mathrm{~kg} / \mathrm{m}^{2} ; p<.0001\right)$, fat-free mass $(36.3 \pm 8.3$ vs. $39.5 \pm 6.0 \mathrm{~kg} ; p<$ $.01)$, fat mass $(23.7 \pm 9.1$ vs. $26.8 \pm 7.2 \% ; p<.05)$, and RMR $(5.9 \pm 0.7$ vs. $6.1 \pm 0.5 \mathrm{MJ} / \mathrm{d} ; p<.05)$ compared to the group with highest SI, whereas in boys no significant group differences were found for these parameters $(p>.05)$. In boys only significant differences between the lowest and highest tertile of SI were obtained for PAL $(1.49 \pm 0.12$ vs. $1.56 \pm 0.14 ; p<.01)$ and duration of sports $(69 \pm 82$ vs. $93 \pm 110 \mathrm{~min} / \mathrm{d}$; $p<.05)$. No significant group differences were found for food intake and BoneHEI in both girls and boys ( $p>.05$ ). Girls with a low body mass, BMI, fat-free mass, fat mass, and RMR and boys with a low physical activity behavior are at high risk for low SI. We recommend school children to perform regular physical activity in order to improve their bone status.

\section{Diagnostic Utility of Total and Free Fraction of Hydroxyvitamin D in} Assessing of Vitamin D Status in Healthy Professional Male Athletes

J Malczewska-Lenczowska $^{1}$, O Surała ${ }^{1}$, D Sitkowski ${ }^{2}$, B Szczepańska ${ }^{1}$, M Ozimek ${ }^{2}$

${ }^{1}$ Department of Nutrition Physiology and Dietetics, Institute of Sport, National Research Institute, Warsaw, Poland

${ }^{2}$ Department of Physiology, Institute of Sport, National Research Institute, Warsaw, Poland

In the athletic population, it is not clear whether the evaluation of serum $25(\mathrm{OH}) \mathrm{D}$ concentrations is sufficient to precisely identify the vitamin D status. It is hypothised that physical effort may induce the acute phase reaction, which may periodically decrease vitamin $\mathrm{D}$ protein binding and reduce a total $25(\mathrm{OH}) \mathrm{D}$ concentration. Recently, an unbound bioavailable fraction of $25(\mathrm{OH}) \mathrm{D}$ (free fraction) was proposed to assess vitamin $\mathrm{D}$ status in physically active subjects. The study aimed to compare diagnostic utility of two metabolites of vitamin D, i.e., total- and free $25(\mathrm{OH}) \mathrm{D}$, in professional athletes subjected to daily training. The study included 131 healthy males, 97 athletes and 34 non-active subjects, aged $17 \pm 1$ and $18 \pm 1$ y respectively. In the fasted, morning blood samples, the following indices were assessed; in serum: total- and free 25(OH)D (ELISA, 
DiASource), C-reactive protein (CRP) concentrations, and creatine kinase activity (CK); in whole blood: leukocytes count (WBC) and erythrocyte sedimentation rate (ESR). Association between two continuous variables was assessed with the Mann-Whitney U test. The correlations between variables were analysed with the Pearson $r$ correlation. The mean concentrations of total- and free $25(\mathrm{OH}) \mathrm{D}$ were higher in the athletes than the control group (total $25(\mathrm{OH}) \mathrm{D}$ : $27.9 \pm 6.7 \mathrm{ng} / \mathrm{ml}$ vs. $20.0 \pm 4.2 \mathrm{ng} / \mathrm{ml}$, $p<.000$; free $25(\mathrm{OH}) \mathrm{D}: 7.5 \pm 2.5 \mathrm{pg} / \mathrm{ml}$ vs. $5.2 \pm 1.6 \mathrm{pg} / \mathrm{ml}, p<.000$, respectively). The indices of the acute phase reaction were in normal ranges in all subjects. The relationship between total- and free $25(\mathrm{OH}) \mathrm{D}$ was strong (athletes: $\mathrm{r}=0.863$; control group: $\mathrm{r}=0.913$ ) and comparable in both groups $(p=.260)$. In athletes, both fractions of 25(OH)D did not differ in the subgroups with normal $(\leq 190 \mathrm{U} / \mathrm{L})$ and with increased (>190 U/L) CK activity, and amounted: total 25(OH)D: $27.6 \pm 6.5 \mathrm{ng} / \mathrm{ml}$ vs. $28.2 \pm 6.9 \mathrm{ng} / \mathrm{ml}(p=.628)$; free $25(\mathrm{OH}) \mathrm{D}: 7.4 \pm 2.7 \mathrm{pg} / \mathrm{ml}$ vs. $7.5 \pm 2.3 \mathrm{pg} / \mathrm{ml}(p=.482)$. In conclusion, the presented results indicate that daily physical efforts do not significantly affect a total $25(\mathrm{OH}) \mathrm{D}$ concentration in healthy professional athletes. It seems that in athletes without acute-phase reaction symptoms, a total $25(\mathrm{OH}) \mathrm{D}$ concentration is an equally good indicator of vitamin D status as the free fraction.

\section{Predicted Versus Actual Metabolic Rates in Elite Level Boxers Continuously 'Making Weight'}

\section{T Simper ${ }^{1}$, A Chappell ${ }^{2}$, M Ellison ${ }^{3}$}

${ }^{1}$ Sheffield Hallam University, Department of Food and Nutrition, Pond Street, Sheffield South Yorkshire, S1 1WB, United Kingdom

${ }^{2}$ Robert Gordon University, School of Pharmacy and Life Science, Robert Gordon University, Garthdee Road, Aberdeen, AB107AQ, United Kingdom

${ }^{3}$ GB Boxing, English Institute of Sport, Coleridge Road, Sheffield, S95DA

Chronically suppressed energy intake is known to effect resting metabolic rate (RMR) which may have long and short-term negative consequences for health including, future weight gain, impaired immunity, and cognitive function. The chronic suppression of weight/energy intake is also associated with relative energy deficiency syndrome. We wished to know if, and to what extent, elite fighters' RMR was suppressed due to chronic energy restriction for the purpose of continuously 'making weight'. Knowledge around any disparity between predicted and actual RMR may be helpful for athletes and coaches in protecting the current and future health of elite boxers. 9 Elite level boxers ( 2 female) $21 \pm 2$ years old were measured in our laboratory whilst preparing for the 2018 Commonwealth games. We carried out anthropometric observations including height, mass and RMR using established methods of good practice. RMR was then compared to three BMR prediction equations. BMR prediction equations over estimated energy requirements in 6/9 fighters (see table and figure 1). Mean RMR values (1407 Kcals) were $299 \pm 417 \mathrm{kcal}$ lower than predicted values from three validated equations. Mean measured RMR was lower than predicted BMR, although there was variation between subjects. Prediction equations are known to typically overestimate BMR, but the difference seen here was typically $>10 \%$. These lower values may therefore be indicative of metabolic adaptations associated with chronic low energy availability. Consideration should be given to both the long-term and short-term health consequences of athletes trying to maintain low body mass.

\section{Body Composition of Female and Male Rowers in Traditional Rowing (Fixed Bank Rowing)}

\section{H Albizu-Yeregui}

University of Basque Country (UPV-EHU), Spain

An adequate body composition is a fundamental variable in the control of sports performance. The objective of this study was to measure the anthropometric parameters of female and male rowers in fixed bank, in order to know the different body masses and body index and the difference between sexes, in addition to the correlation between different variables and to compare the different formulae. This research was a descriptive cross-sectional study and included 73 rowers (52 male, 21 female). The anthropometric measurements (body mass, height, seated size, wingspan, 8 bone diameters, 11 skinfolds and 13 perimeters) were taken following the International Society for Advancement of Kinanthropometry (ISAK) protocol and by using bioelectrical bioimpedance (BIA). The fat, muscle, bone, residual and skin mass was calculated using different methods: fractionation of 5 components, prediction formulae (Carter and Faulkner for fat mass, Martin and Lee for muscle mass, and Roche for bone mass) and bioimpedance (for muscle mass and fat mass). The components of the somatotype were obtained by the Heath \& Carter equation. A body fat percentage of $8 \%$ vs. $18 \%$ (men vs. women) was observed according to the Carter equation, $11 \%$ vs. $20 \%$ according to Faulkner and $12 \%$ vs. $25 \%$ according to BIA. Regarding muscle mass, $33 \%$ vs. $23 \%$ according to Lee, $43 \%$ vs. $31 \%$ according to Martin and $37 \%$ vs. $27 \%$ according to BIA. When referring to the results of the Bland-Altman statistic it is observed that when comparing the different formulae in pairs they had an almost perfect or very good correlation of Pearson but not a good concordance. In men, a mesomorphic somatotype stands out (2.3-5.0-2.7) while in women the endo-mesomorphic one stands out (4.3-4.1-2.0). The male and female rowers have more muscle mass (men have more) and less fat (men has less) than the reference values, according to Phantom's proportionality model, positive data for the anthropometry of an athlete. Although they follow the same pattern, there is a variety of anthropometric profiles, suggesting that different anthropometric compositions may be needed to balance the boat according to the different conditions of the regattas. The different formulae used have good correlation but not good agreement.

\section{Risk of Low Energy Availability in Elite Male Athletes with Spinal Cord Injury}

S Glasgow, K Pritchett, T Stellingwerff, E Broad, R Pritchett, D Ogan

Department of Health Sciences, Central Washington University, United States

Recent research examining male able-bodied athletes has suggested that males are at risk for low energy availability (LEA); however, there is a paucity of research pertaining to prevalence amongst male athletes with an impairment. Therefore, the purpose of this study was to examine the risk of LEA and prevalence of symptoms related to Relative Energy Deficiency in Sport (RED-S), including risk of disordered eating, hormonal profiles, and bone mineral density (BMD) scans, in male wheelchair para athletes. Collegiate- and national-level male athletes $(n=9)$ with spinal cord injury (SCI) completed 7-day concurrent diet and training logs, Eating Disorder Examination Questionnaire (EDE-Q), Dual Energy X-Ray Absorptiometry (DXA) scans, and hormonal blood spot testing. Zero (0) athletes were considered "at-risk" for LEA via EDE-Q score. Useable data to calculate energy availability (EA) was only available for 4 athletes, none of which presented with LEA (using a threshold of $\leq 25 \mathrm{kcal} \cdot \mathrm{kg}$ FFM-1.day-1). Testosterone was low in $100 \%$ of athletes (mean: $7.9 \pm 2.3 \mathrm{nmol} / \mathrm{L}$ ). $25 \%$ of subjects had clinically low hip Z-scores (Z-score $<-2$ ), and $63 \%$ of subjects were at an increased risk for fracture $(Z$-score $<-1$ ). Based on the EDE-Q and calculated EA, the risk for LEA appears to be low; however, the risk for LEA appears to be high based on DXA scans and hormonal profiles. These results are consistent with literature examining LEA in able-bodied athletes, which shows calculated EA to be a poor field assessment tool for LEA. These results also underscore the need for additional research to establish para athlete-specific thresholds for LEA, assessments for disordered eating, and normal clinical hormone ranges. 